\title{
Trauma et hantise de soi dans The Mai et By the Bog of Cats de Marina Carr
}

\section{Hélène Lecossois}

\section{Q OpenEdition \\ 12 Journals}

\section{Édition électronique}

URL : http://journals.openedition.org/etudesirlandaises/2206

DOI : 10.4000/etudesirlandaises.2206

ISSN : 2259-8863

Éditeur

Presses universitaires de Rennes

\section{Édition imprimée}

Date de publication : 30 juin 2011

Pagination : 147-159

ISBN : 978-2-7535-1348-8

ISSN : 0183-973X

Référence électronique

Hélène Lecossois, "Trauma et hantise de soi dans The Mai et By the Bog of Cats de Marina Carr ", Études irlandaises [En ligne], 36-1 | 2011, mis en ligne le 30 juin 2013, consulté le 01 mai 2019. URL http://journals.openedition.org/etudesirlandaises/2206; DOI : 10.4000/etudesirlandaises.2206

Ce document a été généré automatiquement le 1 mai 2019.

(c) Presses universitaires de Rennes 


\title{
Trauma et hantise de soi dans The Mai et By the Bog of Cats de Marina Carr
}

\author{
Hélène Lecossois
}

The Mai et By the Bog of Cats ${ }^{1}$ explorent le côté sombre de la maternité qu'elles lient pour l'une au matricide, à l'infanticide et au suicide, pour l'autre au suicide uniquement. La mort occupe ainsi une place essentielle, aussi bien sur le plan thématique que structurel ou formel. La fable s'articule autour de la perte qu'ont subie les deux protagonistes et de la mort que Mai et Hester se donnent. Contrairement à By the Bog of Cats, qui confronte le spectateur directement à la mort violente de l'héroïne (et au meurtre de sa fille), The Mai lui épargne la vue du suicide, qui a lieu hors scène. Le corps mort de Mai n'en est cependant pas moins exhibé à la fin du premier des deux actes que compte la pièce. La place médiane qu'occupe la monstration du cadavre dans l'économie générale de l'œuvre signale la centralité de la mort ${ }^{2}$, tout en remettant en question le caractère définitif et irrévocable de celle-ci - le second acte revenant sur un moment précédant le suicide. La prosopopée inscrit le passé dans le présent et nie toute solution de continuité entre la vie et la mort. À une structure temporelle linéaire et orientée, Marina Carr préfère une construction analeptique, un entrelacs de présent et de passé et une cyclicité. La temporalité qu'elle instaure est celle du retour et de la répétition, dont on sait depuis Freud qu'ils sont symptomatiques du trauma. La nature inassimilable de celui-ci force, en effet, le sujet, en proie à des forces autres que sa volonté (Freud parle de «destin»), à revivre l'expérience traumatisante. Ecartelés entre un passé qui ne passe pas et ne cesse de faire retour et un avenir qui n'offre aucune perspective de changement, les personnages de Carr semblent condamnés à éternellement revivre ou rejouer la même histoire et à (re)faire continuellement l'expérience de la perte et de la dissolution. L'une des principales caractéristiques des protagonistes de The Mai et By the Bog of Cats est leur spectralité : Mai et Hester sont marquées du sceau d'une mort prochaine ou déjà advenue. Fantomatiques, elles sont elles-mêmes hantées par d'autres fantômes qui les rongent de l'intérieur. Cependant, s'ils les vident effectivement de leur substance, ces fantômes «encryptés ", pour le dire avec Nicolas Abraham et Maria Torok ${ }^{3}$, leur confèrent 
également une certaine densité en faisant d'elles le lieu où viennent s'accumuler, tels des sédiments, les histoires d'autres femmes. Les personnages apparaissent comme un mélange paradoxal d'insubstance et d'excès de présence(s). The Mai et By the Bog of Cats s'efforcent de représenter, c'est du moins ce que cet article se propose de démontrer, le trauma de sujets dont l'avènement n'a jamais vraiment eu lieu, de sujets condamnés à vivre dans la hantise de l'individuation.

2 The Mai et By the Bog of Cats nous donnent à voir un feuilleté temporel dont les strates sont peuplées d'êtres fantomatiques. L'immatérialité spectrale des personnages déjà morts de The Mai est redoublée par l'aspect fantomatique qu'ils avaient déjà de leur vivant : lorsque Mai se prépare pour accompagner Robert au bal du Lion's Club, elle suscite les commentaires suivants: "Beck: Mai, you're a picture. / Millie: You are, Mom. / Grandma Fraochlán: An apparition if ever I saw one » (166). La superbe robe de soirée dont Mai s'est parée ne réussit pas à masquer son absence de substance, qui est, au contraire, mise en valeur. Le sens littéral de l'expression laudative vient se superposer au sens métaphorique. Privée de toute épaisseur, Mai est ainsi comparée à un tableau ou à une image. Le regard aiguisé de Grandma Fraochlán, opiomane invétérée, experte dans l'art de la fantasmagorie, ne s'y trompe pas, et le mot "apparition » insiste sur l'aspect spectral de Mai. La première scène de By the Bog of Cats nous confronte à une ombre protatique, le Ghost Fancier, dont les propos nous annoncent que la scène sera et est déjà peuplée de fantômes: «[...] Where there's ghosts there's ghost fanciers" (265). Même si Hester lui signifie son erreur, en lui disant que ce ne peut être elle qu'il est venu chercher puisqu'elle est encore en vie («I certainly am [alive] and aim to stay that way » (266)), elle est déjà marquée du sceau d'une mort prochaine. Au fil des scènes, sa nature spectrale est d'ailleurs de plus en plus perceptible ; elle-même déclare à l'acte III : «[...] For a long time now I been thinking I'm already a ghost » (321). Dans son étude sur les fantômes épiques et tragiques, Patricia Vasseur-Legangneux nous explique que, chez Homère, l'eidôlon est « un corps vidé de sa substance et de sa force. Le terme d'ombre est alors employé (skia) pour qualifier ce corps qui a perdu son principe vital, le thumos, est capable de s'envoler [...] et est comparé à une fumée ou à un oiseau dont il peut avoir le cri aigu ${ }^{4} »$. Le cygne noir, présenté comme l'ombre d'Hester dans la première scène, ("What're you doin' draggin' the corpse of a swan behind ya like it was your shadow? » $(265)^{5}$, lui demande le Ghost Fancier) fait retour, lors de la scène finale, qui nous donne à voir son cœur, métamorphosé en cygne : "She's cut her heart out - it's lyin' there on top of her chest like some dark feathered bird» (341). L'image du cygne rattache incontestablement Hester à la mythologie celtique, elle fait également écho aux fantômes épiques, d'autant qu'Hester pousse un long cri animal peu avant de mourir.

3 À tous ces spectres qui peuplent la scène de ce théâtre d'ombres, s'ajoute une sorte de méta-fantôme, celui du personnage de théâtre. Les masques que nous donne en effet à voir la mimésis théâtrale font du personnage une présence-absence dont la nature ne diffère pas fondamentalement de celle du fantôme. Cela est d'autant plus vrai chez Marina Carr que les personnages auxquels elle donne naissance portent plusieurs masques et qu'ils conjoignent donc plusieurs présences-absences: Hester Swane est un avatar de Médée, Mai une réincarnation de Coillte, personnage de légende dont Millie rapporte l'histoire à la fin de l'acte I. L'irlandicité de ce nom, comme le localisme que Marina Carr fait entrer dans la langue, en reproduisant au plus près l'accent plat et guttural des Midlands - cette région dont le nom même et l'emplacement géographique signalent l'entre-deux et reflètent le caractère liminal des personnages qui l'habitent, fait 
surgir un dernier fantôme, celui de la langue. Le gaélique hante la scène de The Mai : la phrase qui ensorcelle Grandma Fraochlán et la fait succomber aux charmes du " pêcheur aux neuf doigts » a été prononcée en gaélique ${ }^{6}$ et des termes tels que « a stóir » ou " a chroi " ponctuent le discours des personnages. Cette langue qui affleure régulièrement à la surface du texte, qui fait retour et hante l'autre langue, est liée à l'affect et au regret. Elle peut être entendue comme l'expression d'un deuil qui ne se fait pas. Elle signifie aussi que la «vraie » langue est ailleurs; exilé dans la langue, le sujet n'a de cesse que de se heurter à l'indicible. La " fantômachie ", pour reprendre le terme de Derrida, que Marina Carr nous donne à voir nous invite à réfléchir sur la nature de la présence-absence qui constamment fait retour sur scène. Nous interprétons la spectralité des personnages et de la langue comme autant de manifestations symptomatiques d'un trauma. Le spectre, entité indécise qui allie des réalités contradictoires telles que présence et absence, évanescence et rémanence, donne corps, pour ainsi dire, au trauma en ce qu'il signale la présence insistante de l'invisible, de ce qui reste hors de portée de la conscience du sujet - de ce qui, en d'autres termes, lui crève les yeux, au sens propre et au sens figuré.

4 La dramaturgie de la revenance que Marina Carr met en place évoque par bien des aspects un travail de deuil qui n'aboutit pas. Sa poétique du deuil impossible s'appuie sur une dichotomie de l'excès et du manque. L'exemple le plus frappant est sans doute celui de la rame de Curragh dans The Mai. Relique du défunt mari de Grandma Fraochlán dont celle-ci ne se déleste jamais, les proportions exubérantes de cette rame contraignent les personnages à redessiner les contours de l'espace qu'ils habitent («Connie: We had to saw through the banister to get it into our house ", 113) et signalent le caractère envahissant de la mort qui ne laisse que peu d'espace aux vivants. Le gigantisme de la rame fait écho à celui de la fenêtre de la maison de Mai dont Connie dit qu'elle n'en a jamais vue de plus grande. La taille de la rame et celle de la fenêtre sont à la mesure de la perte qu'ont subie Grandma Fraochlán et Mai. La matérialité envahissante de l'une et la béance qu'encadre l'autre figurent toutes deux le vide ou le deuil impossible et dessinent les contours de l'espace du trauma. Les morts ou leurs fantômes occupent le centre de l'espace scénique et envahissent l'espace psychique des protagonistes, les laissant dans l'incapacité de choisir quelque nouvel objet d'amour.

5 Le deuil impossible dans lequel les personnages sont figés rejaillit sur les paysages qu'ils habitent et leur donnent des accents mélancoliques certains. Le spectateur est ainsi confronté à une surabondance de signes connotant la mort, l'absence, le vide et est frappé par la dimension létale de l'univers qui se dévoile. Dans By the Bog of Cats le rideau se lève sur un paysage lugubre et enneigé qu'un cadavre de cygne noir vient maculer de sang et sur la mention d'une tombe qu'Hester s'apprête à creuser. The Mai s'ouvre sur le retour, après de longues années d'absence, de l'être aimé, mais n'en demeure pas moins une pièce de la perte, ce que dit clairement le caractère élégiaque de la narration de Millie. La nature mortifère des signes exposés au tout début de la pièce ne sera cependant perçue qu'a posteriori : le rêve prémonitoire que raconte Robert dans la première moitié de l'acte I transforme les cygnes, l'étui de violoncelle et le lac sur lequel s'ouvre la fenêtre en autant de signes annonciateurs de mort.

Robert - I dreamt that you were dead and my cello case was your coffin and a carriage drawn by two black swans takes you away from me over a dark expanse of water and I ran after this strange hearse shouting, "Mai, Mai" [...] 
The Mai - So you've come back to bury me, that what you're sayin'?

(The Mai, 125)

6

La maison près d'Owl Lake est, du dire même de Mai, de celles que l'on construit quand on n'a plus nulle part où aller. « Millie : The Mai set about looking for that magic thread that would stitch us together again and she found it at Owl Lake, the most coveted site in the country » (111). La métaphore du fil, la ressemblance entre Mai, femme fidèle attendant patiemment le retour de son mari parti dans une contrée lointaine, et la Pénélope de la mythologie grecque nous invitent à voir la maison comme un avatar du linceul de Laerte que Mai tisse/bâtit pour gagner du temps. Dans l'espace scénique et dramatique de The Mai et By the Bog of Cats, les signes mortifères se conjuguent à ceux qui figurent la vacuité. L'une des images autour desquelles se structurent les deux pièces est celle de la béance. La mort s'inscrit littéralement en creux sur la surface de la tourbière. C'est dans un trou que Catwoman voit, malgré ses yeux aveugles, la tragédie à venir («Catwoman : Lave this place now or you never will./Hester : I'm stoppin' here./Catwoman : Sure I know that too. Seen it writ in a bog hole », 276-277). C'est dans un trou également qu'Hester Swane retrouve le corps gelé du cygne noir, son double métaphorique. La tourbière toute entière apparait ainsi comme une tombe ouverte, à l'image de celle que creuse Hester pour Black Wing. Dans The Mai, c'est la fenêtre de la maison-linceul qui figure la béance et rend manifeste l'invisible - que celui-ci prenne la forme de la légende tragique que Mai revit malgré elle ou de l'espace qui ne sera jamais offert à la vue des spectateurs, le hors scène, que la mention des différents rêves nous invite à appréhender comme lieu de l'inconscient. Donnant sur Owl Lake, dont la légende dit qu'il s'est formé des pleurs de Coillte et dans lequel celle-ci meurt noyée, la fenêtre est moins ouverture sur un extérieur ou un ailleurs que miroir confrontant Mai à ses doubles spéculaires. La scène s'apparente à bien des égards à une caisse de résonnance dans laquelle les histoires des différentes générations de femmes que The Mai met en scène se font écho, comme autant de variations sur le même thème. La matrice, que les histoires de Millie, Mai, Ellen ou Grandma Fraochlán ne font que dupliquer, est la légende de Coillte et Bláth qui ellemême, peut se lire comme une version irlandaise du mythe de Déméter et Perséphone ou de celui d'Eurydice et d'Orphée.

7 Le paysage de désolation, de tristesse infinie et de mort sur lequel donne la fenêtre duplique l'intérieur de la maison, lui-même espace de chagrin inconsolable. Intérieur et extérieur en viennent à se confondre. L'espace scénique et dramatique se fait espace psychique et relève d'une géographie de l'intime et du subjectif. Marina Carr construit une véritable poétique de la mélancolie en mettant en scène des héroïnes qui font littéralement corps avec un espace mortifère. «Millie: [...] Heartbroken Coillte lay down outside the dark witch's lair and cried a lake of tears that stretched for miles around. One night, seizing a long-awaited opportunity, the dark witch pushed Coillte into her lake of tears » (147). Le lac s'origine dans le corps de Coillte; il est aussi le lieu de sa dissolution. Hester Swane, comme Coillte-Mai, s'inscrit dans un rapport de consubstantialité avec le paysage. Elle ne se sent chez elle que lorsqu'elle arpente la tourbière avec laquelle elle fusionnera après sa mort :

Hester - Ya won't forget me now, Carthage, and when all of this is over or half remembered and you think you've almost forgotten me again, take a walk along the Bog of Cats and wait for a purlin' wind

Études irlandaises, 36-1 | 2011 
through your hair or a soft breath be your ear or a rustle behind you.

That'll be me and Josie ghostin' ya. (340)

8

La représentation du trauma passe chez Marina Carr par la mise en texte de répétitions compulsives. Dans By the Bog of Cats, la tourbière est le lieu où se rejoue inlassablement le départ de la mère, qui, par deux fois, interdit à sa fille de la suivre et la condamne à la regarder s'éloigner, impuissante :

\begin{abstract}
Hester - [...] And she says, "I'm goin' walkin' the bog, you're to stay here, Hetty." And I says, "No", I'd go along with her, and made to folly her. And she says, "No, Hetty, you wait here, I'll be back in a while." And again I made to folly her and again she stopped me. And I watched her walk away from me across the Bog of Cats. And across the Bog of Cats I'll watch her return. (297)
\end{abstract}

Ce qui préside par la suite aux actions d'Hester, c'est la répétition infinie de cette scène traumatisante. Son errance compulsive reproduit celle de sa mère et le meurtre de sa fille peut être perçu comme l'actualisation de ce que sa mère lui a fait subir symboliquement. Hester assimile, en effet, l'abandon de sa mère, et le regard qu'elle lui jeta en s'éloignant, à un meurtre, comme le signale le verbe "do away» :

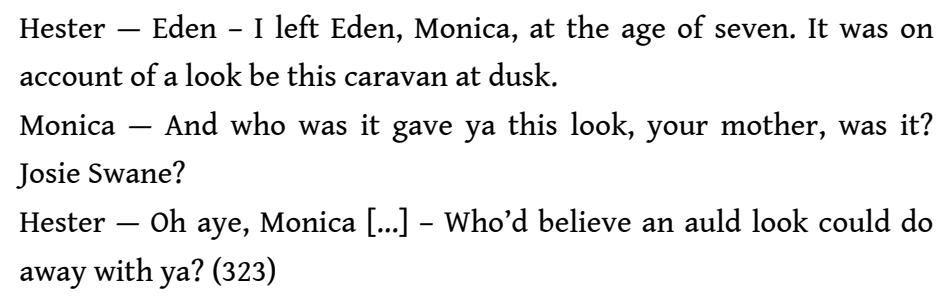

10 Le regard de Josie Swane, aussi terrible que celui de Méduse, laisse sa fille à jamais sidérée. L'événement traumatique est soustrait à la temporalité et ne trouve pas d'ancrage, de lieu psychique, dans le temps subjectif d'Hester qui, à presque quarante ans, croit encore aux contes de fées et continue à espérer le retour de sa mère ${ }^{7}$. Lorsque Millie évoque le moment où sa mère travaillait à Londres et était devenue la coiffeuse attitrée d'une petite princesse arabe, elle établit une analogie entre Mai et cette petite princesse :

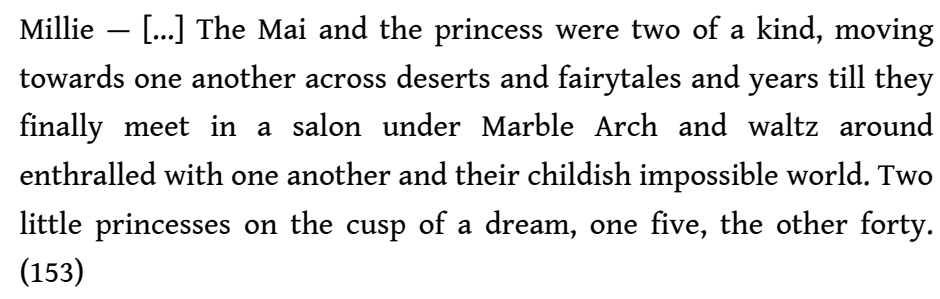

11 Cette histoire rappelle celle de Grandma Fraochlán qui se fantasme en personnage de légende et s'imagine être la fille du Sultan d'Espagne. Les héroïnes de Marina Carr ressemblent à des matriochkas: doubles de figures mythologiques, elles donnent naissance à d'autres doubles et sont rongées de l'intérieur par un vide qu'elles sont impuissantes à combler. Les histoires de princesses, de sultan ou de mère qui reviendra un jour près de la "tourbière des chats ", ne font que redire ce vide insondable. Dans la réponse qu'elle fait à Grandma Fraochlán, Julie sous-entend l'incapacité de l'excès à masquer le manque : 
Grandma Fraochlán - I told ya, ya eejit, my great grandfather was Tunisian! I'm only quarter Tunisian, half Maroccan and half Spanish! Julie - That makes five quarters! How many quarters in a whole?

12 La prolifération des fantasmes vide la réalité de toute substance. Celle des spectres annihile le présent. Présence du passé qui ressurgit dans le présent, le spectre brouille les repères temporels. Les personnages hantés de The Mai et de By The Bog of Cats portent en eux le signe que la mort habite le vivant, que la catastrophe a déjà eu lieu et qu'elle aura lieu à nouveau. Cette catastrophe à la fois passée et future vide le présent de sa substance. Le présent de l'indicatif signale alors une temporalité à jamais figée, celle de l'éternel retour. Dans By the Bog of Cats le temps de la hantise est signalé par l'erreur du Ghost Fancier qui confond l'aube et le crépuscule. Cette aube crépusculaire est le moment où le cours du temps s'inverse, le passé devenant futur et le futur passé. Le futur antérieur apparaît donc aussi comme le temps de la hantise chez Marina Carr. Dans « Théorie de la hantise ", Jean-Jacques Lecercle pose que «la hantise brouille l'espace (en ce qu'elle n'admet ni incarnation ni objectivation) ", la hantise est, en effet, une expérience subjective, l'intuition de ce qui va advenir au sujet mais qui n'a pas de réalité pour lui, à savoir sa propre mort. Elle brouille aussi « le temps (en ce qu'elle illustre le paradoxe d'un avenir qui laisse des traces rétrospectives dans le présent : la hantise est [donc] une forme d'anachronie) ». Jean-Jacques Lecercle explique que :

Le spectre existe dans l'espace, autant que dans le temps, ce qui est normal si c'est un objet. Et la hantise, ce qui est normal pour un procès, n'existe que dans le temps. Mais ce temps n'est pas le même. Le temps du spectre est le passé, celui de la hantise l'avenir. Si on vit dans la hantise de sa propre mort, c'est dans l'avenir que la hantise situe l'objet qu'elle vise, et c'est dans l'avenir qu'elle transporte le sujet qui l'éprouve ${ }^{8}$.

13 L'aube crépusculaire sur laquelle s'ouvre By the Bog of Cats est intéressante en ce qu'elle conjugue le crépuscule, moment où se réveillent traditionnellement les morts qui viennent hanter les vivants, et l'aube qui porte les traces rétrospectives de la mort à venir. Dans The Mai, la présence-absence de la mort à la fois analeptique et proleptique, fait du présent de l'histoire un entrelacs d'éternel retour et de hantise. La fenêtre s'ouvre sur le présent a-temporel de la mort du personnage légendaire de Coillte et sur le futur antérieur de la mort de Mai : la prosopopée transformant la mort passée en une mort à venir. Lorsque Robert, qui dit avoir rêvé de façon récurrente de la mort de Mai, lui demande si elle n'a jamais rêvé de sa mort à lui, voici ce qu'elle lui répond :

I dreamt it was the end of the world and before my eyes an old woman puts a knife through your heart and you die on the grey pavement, and for some reason I find this hilarious. Then the scene changes and I'm a child walking up a golden river [...] At the bend in the river I see you coming towards me whistling through two leaves of grass - you're a child too - and as you come nearer I smile and wave, so happy to see you, and you pass me saying, "Not yet, not yet, not for thousands and thousands of years." And I turn to look after you and you're gone and the river is gone and away in the distance I see a black cavern and I know it leads to nowhere and I start walking that way because I know I'll find you there. (126) 

dont on dit, traditionnellement, qu'il hante l'amour maternel. Dans cette pièce où le cours $\mathrm{du}$ temps peut s'inverser et où les mères et les filles se fondent en une figure indifférenciée, l'infanticide fait surgir le spectre du matricide. Hester fantasme, d'ailleurs, le meurtre de sa mère - "If she showed up now I'd spit in her face, I'd box the jaws off of her, I'd go after her with a knife » (318). Le meurtre symbolique n'est pas pour autant accompli. On pourrait penser qu'il l'est, à la toute fin de la pièce, et qu'Hester actualise son fantasme lorsqu'elle retourne le couteau contre elle-même. A ce moment-là, Josie apparaît comme le double d'Hester enfant - elle a l'âge qu'avait Hester au moment du départ de sa mère et porte, comme Hester à l'époque sa robe de communion - et Hester comme le double de Josie Swane, sa mère. Hester et Josie meurent en prononçant les mêmes mots : « Mam, Mam » et laissent sur la tourbière enneigée une trace ensanglantée qui n'est pas sans rappeler celle qu'avait laissée le cygne noir au début de la pièce. La répétition et le palindrome accentuent l'impression de circularité. Celle-ci signale que la déliaison d'avec 
la mère, le matricide symbolique, qui ouvrirait sur l'individuation et l'altérité n'a pas eu lieu.

Si le rapport entre By the Bog of Cats et son hypotexte, Médée, est évident, celui qui unit The Mai à Electre, l'un des intertextes cités par la critique, l'est moins. Marina Carr met bien en scène des femmes endeuillées dont le père est absent. Il n'est cependant jamais question de vengeance. C'est davantage à la psychologie du personnage d'Electre qu'à l'intrigue de la tragédie de Sophocle, d'Euripide ou d'Eschyle que The Mai fait écho. Electre reproche à sa mère de faire passer son bon plaisir avant le sien et lui tient grief d'être davantage amante que mère. C'est précisément ce qui est reproché à celle qui fait figure de mère archétypale dans The Mai : Grandma Fraochlán. Les femmes de la pièce ne sont pas en manque de mari ou de père, elles sont en manque d'amour maternel. Celui-ci a toujours fait défaut, comme en témoigne, par exemple, le fait que la mère de Grandma Fraochlán la forçait à l'appeler " The Duchess ", la mettant ainsi d'emblée à distance et lui interdisant l'expression de toute marque d'affection. Oreste, que Michèle Gastambide voit comme interchangeable avec Electre en raison du fait que la question du matricide concerne «le sujet dans tout son être, féminin et masculin, indépendamment du pôle sexuel où il a dû se placer" ", hésite avant d'affronter l'ultime tabou que représente le meurtre d'une mère. Voici ce que Michèle Gastambide écrit du temps que se ménage Oreste avant le meurtre dans Le meurtre de la Mère. Traversée du tabou matricide :

Anticipation terrifiante et fascinante de l'orage émotionnel et narcissique qui va embraser son être à l'instant où son bras, d'un seul et même mouvement, empreint d'une toute-puissance vertigineuse, va réaliser la relation la plus proche qui soit avec une mère, la plus mortifère, la plus interdite et du même mouvement, la coupure la plus radicale, la plus libératoire d'avec elle ${ }^{10}$.

Euripide et Sophocle présentent un Oreste qui n'a pas encore triomphé de son apathie. Seul Eschyle, toujours selon les mots de Michèle Gastambide, «ne recule pas devant l'ultime moment de vérité : la rencontre du fils avec la mère où vont se découvrir leurs désirs ${ }^{11} »$.

Dans By the Bog of Cats et The Mai, Marina Carr nous met en présence de femmes incapables d'accomplir le geste libératoire qui célèbrerait la déliaison d'avec la mère. Englués dans des questions d'héritage et de filiations, les personnages sont des êtres dépouillés de toute identité singulière. Leurs désirs ne peuvent se découvrir, étant toujours voilés par celui de la mère. La hantise d'eux-mêmes dans laquelle les personnages féminins vivent, nous la lisons comme la principale manifestation du trauma que ces personnages ont subi. La nature, par essence, inassimilable du trauma empêche toute représentation directe ou frontale. Pourtant, dans les pièces de Carr (cela est vrai d'autres pièces, telles Portia Coughlan ou Womanand Scarecrow, par exemple) la réalité factuelle du trauma est exprimée sans détour. Les personnages semblent parfaitement conscients des maux qui les affectent. Catwoman souligne ainsi qu'Hester est douée d'une clairvoyance et d'un discernement particulièrement aigus : " the way ya go on as if God gave ya a little frog of a brain instead of the gift of seein' things as they are, not as they should be, but exactly as they are » (273-274). Dans son introduction à The Dazzling Dark. New Irish Plays, Frank McGuinness écrit : "Tragedy is so often the consequence of a fatal lack of self-knowledge. Marina Carr rewrites that rule. Her characters die from a fatal excess of self-knowledge ${ }^{12}$ ». Si les faits sont exposés, la langue échoue cependant continuellement à dire l'émotion du trauma et la parole n'a aucun effet libératoire. 
21 Carr met en scène des personnages incapables d'inscrire leur histoire dans l'ordre symbolique. L'histoire de Coillte, qui informe celle de toutes les générations de femmes de The Mai, est celle du silence. Coillte ne dit pas son chagrin, c'est son corps qui prend la parole par le biais des larmes. Incorporée, la peine n'est jamais mise à distance parce que jamais mise en mots. Le personnage de Mai, à l'image de celui de Coillte, est associé au somatique. Au tout début de la pièce, elle place des livres sur une étagère, comme si par ce geste elle se dissociait de l'ordre du symbolique et écartait la possibilité de dire sa propre histoire. Elle n'est alors qu'un corps-instrument: «He taps her shoulder, hip bone, ankle ", "Now he plays the cello bow across her breasts " (107-108). C'est à Millie que revient le soin de dire son histoire. En tentant de rassembler les morceaux épars de l'histoire de sa mère, Millie se conforme a posteriori au rôle que celle-ci lui assigna le jour du départ du père. Ce jour-là, alors qu'elle tentait de noyer son chagrin dans l'alcool, Mai envoya Millie chez le boucher pour y chercher une aiguille et du fil. L'alcool que Mai avale, comme les larmes que verse Coillte et qui finissent par l'avaler, signalent un besoin de remplir une bouche vide... de mots. Dans la requête impossible à satisfaire que formule Mai se combinent deux images : celle du morcellement, liée à la boucherie, qui fait ressortir l'intensité de l'arrachement que vient de subir Mai et celle du remembrement que signalent l'aiguille et le fil.

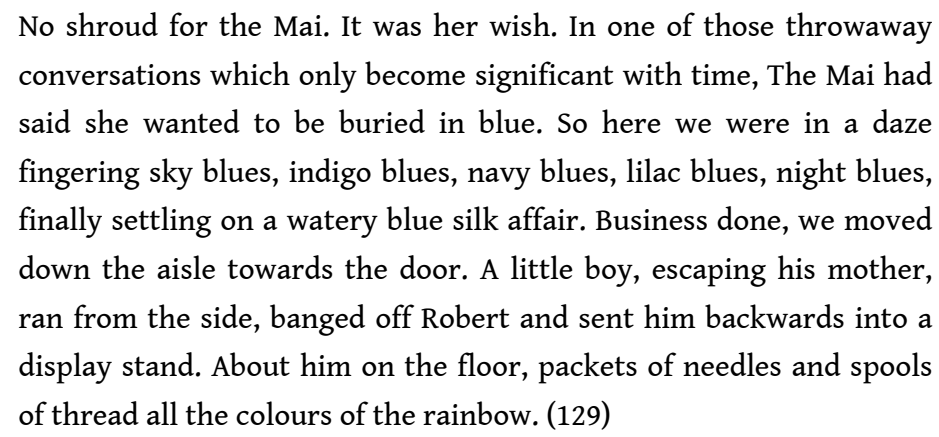

22 La profusion de fils et d'aiguilles signalent que la quête de Millie est arrivée à son terme, ou tout au moins qu'elle va se teinter d'autres couleurs et prendre une autre forme. Munie d'une aiguille et d'un fil métaphoriques, Millie se fait la narratrice de l'histoire de sa mère. D'une main alerte, elle s'efforce d'exorciser la souffrance que celle-ci a endurée et de contenir sa détresse. Millie tente d'offrir aux sujets en défaut d'eux-mêmes que sont son arrière grand-mère, mais surtout sa mère, un cadre pour contenir leur affliction refoulée. Ce cadre est celui de son récit, symbolisé par celui de la fenêtre, près de laquelle elle est constamment postée.

Incapables de dire le trauma, les protagonistes de The Mai et By the Bog of Cats, rejouent à l'infini la scène traumatisante jusqu'à se donner la mort. Cette mort qu'elles se donnent peut, cependant, paradoxalement, se lire comme le signe de leur libération. Dans la lecture qu'il fait de Patočka dans Donner la mort, Derrida écrit ceci :

[...] Faire l'expérience de la responsabilité, [...] c'est faire l'expérience de sa singularité absolue et appréhender sa propre mort, c'est la même expérience : la mort est bien ce que personne ne peut ni endurer ni affronter à ma place. Mon irremplaçabilité est bien conférée, livrée, on pourrait dire donnée par la mort ${ }^{13}$.

The Mai et By the Bog of Cats nous confrontent au paradoxe d'une mort à la fois potentiellement libératrice et aliénante en ce qu'elle ne fait que dupliquer la mort d'une autre. L'aporie devant laquelle la mort des protagonistes nous place est l'une des formes 
que prend la mise en scène du trauma chez Carr. Cependant, la nature somme toute très conservatrice et très logocentrée du théâtre de Carr contribue à tenir à distance la réalité affective et émotionnelle du trauma - par opposition aux théâtres du traumatisme dits " postdramatiques ", celui de Sarah Kane, par exemple, dont tous les composants tendent à représenter l'irreprésentable du trauma. Un hiatus se fait jour entre les thèmes que Carr met au centre de ses pièces et la forme dramatique qu'elle choisit pour celles-ci.

\section{NOTES}

1. L'édition à laquelle les numéros de pages donnés entre parenthèses renvoient est la suivante : Marina Carr, Plays 1, London, Faber and Faber, 1999.

2. Les titres déjà indiquent, même si le spectateur ne le comprend que rétrospectivement, la centralité de la mort : celle du personnage éponyme, Mai, est déjà advenue et c'est près de la « tourbière des chats » que la mort se donne.

3. Nicolas Abraham et Maria Torok, L'écorce et le noyau, Paris, Flammarion, 1987.

4. Patricia Vasseur-Legangneux, «Des fantômes épiques aux fantômes tragiques: héritage, transformations, inventions dans l'antiquité grecque ", Dramaturgies de l'ombre, Françoise Lavocat et François Lecercle (dir.), Rennes, Presses Universitaires de Rennes, collection « Interférences », 2005, p. 16.

5. Les propos du Ghost Fancier font écho aux croyances religieuses concernant les morts dans la Grèce antique : " au terme des rites funéraires, le corps humain revêt la forme d'une réalité à deux faces dont chacune renvoie à l'autre et implique sa contrepartie : une face visible et localisée et une face invisible, insaisissable et fugitive » (J.-P. Vernant cité dans Patricia Vasseur-Legangneux, p. 15). Le mot «corpse » renvoie à la face visible que mentionne J.-P. Vernant, le mot « shadow » à la face invisible.

6. «Grandma Fraochlán: I remember the first time I met the nine-fingered fisherman. 'Is mise Tomás, scipéir, mac scipéara', he said. I knew where he was comin' from, one sentence, one glance of his blue eyes and me heart was in his fist ", The Mai (119).

7. «Monica : You up on forty, Hester, and still dreamin' of storybook endin's, still whingin' for your Mam. » (324).

8. Jean-Jacques Lecercle, «Théorie de la Hantise », Tropismes, $n^{\circ}$ 14, Université de Paris X, 2007, p. 7.

9. Michèle Gastambide, Le meurtre de la Mère. Traversée du tabou matricide, Paris, La Méridienne, 2002, p. 64.

10. Ibid., p. 88.

11. Ibid., p. 89.

12. Franck McGuinness (dir.), The Dazzling Dark. New Irish Plays, London, Faber and Faber, 1996, p. ix.

13. Jacques Derrida, Donner la mort, Paris, Galilée, 1999, p. 64. 


\section{RÉSUMÉS}

Le trauma que The Mai et By the Bog of Cats s'efforcent de représenter est celui de sujets dont l'avènement n'a jamais eu lieu, de sujets condamnés à vivre dans la hantise de l'individuation. La dramaturgie du traumatisme que Marina Carr met en place dans ces deux pièces s'apparente à bien des égards à une dramaturgie de la revenance. Le spectre, entité indécise qui allie des réalités contradictoires telles que présence et absence, évanescence et rémanence, donne pour ainsi dire corps au trauma en ce qu'il fait retour et signale la présence insistante de l'invisible, de ce qui reste hors de portée de la conscience du sujet. La nature par essence inassimilable du trauma empêche toute représentation directe ou frontale. Si, paradoxalement, les pièces de Carr disent la réalité factuelle du trauma sans détour, elles semblent cependant échouer à en exprimer la réalité affective et émotionnelle.

This article aims at defining the nature of the traumatic experience that lies at the heart of Marina Carr's The Mai and By the Bog of Cats. Here, Carr constructs characters that never emerge as subjects. The "subject" staged in the two plays is one whose individuation has never really occurred. The female protagonists, Hester Swane and The Mai, are forever ghosted by their own (m)other. The figure of the spectre, as an indefinite entity which allies contradictory characteristics such as presence and absence, evanescence and persistence, (dis)embodies trauma in that it forever returns, thus signalling the insisting presence of the invisible, of that which forever eludes the subject's consciousness. The essentially inassimilable nature of trauma renders any direct, frontal representation impossible. Whereas Carr's plays allow us to see and to hear the factual reality of trauma, I argue that they also fail to fully express trauma's affective and emotional reality.

\section{INDEX}

Mots-clés : Carr Marina, théâtre, trauma

Keywords : Carr Marina, drama, trauma

\section{AUTEUR}

\section{HÉLÈNE LECOSSOIS}

Université du Maine, Le Mans 